\title{
Impacto del Pinus ponderosa sobre la acidificación de los suelos de la transición bosque-estepa, SO del Neuquén, Argentina *
}

\author{
Impact of Pinus ponderosa on acidification at forest-steppe \\ transition soils, SW Neuquén, Argentina \\ BROQUEN P., CANDAN F., FALBO G., GIRARDIN J.L., PELLEGRINI V. \\ A.U.S.M.A.-F.C.A.-U.N. Comahue, Pasaje de la Paz 235, (8370). S. M. Andes, Argentina. \\ Email: pbroquen@yahoo.com
}

\begin{abstract}
SUMMARY
Soil reaction and some related properties were studied in twenty-four plots approximately distributed through a west-east transverse, twelve under shrub-gramineous steppe, and twelve under Pinus ponderosa Dougl. -first plantation from 14 to 19 years- with equivalent site conditions and whose soil parent material was holocenic volcanic ash under xeric hydric regime. Composite soil samples were analyzed for each centimeter within the five first ones. Soil solution acidity and exchangeable acidity increased under pines in relation with shrub-gramineous steppe vegetation. Acidification was due to the lower buffering capacity of soils under xeric regime and did not meant a change in soil acidity class, being slightly acid under both vegetation types. It can be stated that the rate of acidification within the first five soil centimeters, would not have grate consequences on germination and growth for the autochthonous species and as for futures afforestations because the acidity class, total exchangeable bases, phosphate retention did not change under pines. Soil acidification and exchangeable acidity increment, should be considered as indicators of changes that begun to happen after the afforestations and must be used on future monitoring.
\end{abstract}

Key words: acidification, shrub-gramineous steppe, ponderosa pine, andinopatagonia.

\section{RESUMEN}

Se estudió la reacción del suelo y propiedades relacionadas en veinticuatro parcelas distribuidas en un transverso con una dirección aproximada oeste-este, doce bajo estepa subarbustivo-graminosa y doce bajo Pinus ponderosa Dougl. -primera plantación, 14 a 19 años-, en condiciones de sitio equivalentes y cuyo material parental fue ceniza volcánica holocénica bajo régimen hídrico xérico. Se analizaron muestras compuestas para cada centímetro en los primeros cinco centímetros del suelo mineral. La acidez de la solución del suelo y la de intercambio aumentaron en los cinco primeros centímetros bajo P. ponderosa. La acidificación se debió al menor poder tampón de los suelos bajo régimen xérico y no significó un cambio en la clase de acidez del suelo, manteniéndose como ligeramente ácidos. Dado que bajo P. ponderosa no cambiaron la clase de acidez, el total de bases intercambiables y la retención de fosfato, el grado de acidificación de los primeros centímetros del suelo no generaría variaciones relevantes en las condiciones del suelo para la germinación y crecimiento tanto de las especies autóctonas como de futuras forestaciones. La acidificación e incremento de la acidez intercambiable deben considerarse como indicadores de cambios que comenzaron a ocurrir en el suelo después de la instalación de las forestaciones, debiendo considerar su uso en futuros monitoreos.

Palabras clave: acidificación, estepa subarbustivo-graminosa, pino ponderosa, andinopatagonia.

* Subsidiado por la Secretaría de Investigación de la Universidad Nacional del Comahue. 


\section{INTRODUCCION}

La actividad forestal en la región andinopatagónica, en particular en la provincia del Neuquén, ha determinado el reemplazo parcial de áreas con vegetación nativa de estepa subarbustivograminosa por forestaciones de coníferas exóticas, principalmente con Pinus ponderosa Dougl. En las zonas no forestadas el principal uso agrícola de la tierra es la ganadería extensiva ovinocaprina, donde el sobrepastoreo contribuye al proceso de desertificación. Estimaciones de la erosión a través de mediciones directas, conjuntamente con la estimación del peligro de erosión bajo uso ganadero y forestal simulado con $P$. ponderosa, mostraron el fuerte impacto actual y potencial de las forestaciones sobre el control de la erosión y por ende de la desertificación en la transición bosque-estepa, acercándose a un uso sustentable del sistema (1). En este sentido es importante considerar otras variables que puedan incidir en la sustentabilidad del sistema, tal como la reacción del suelo. Numerosos procesos son afectados por la reacción del suelo y viceversa, entre otros, la tasa de descomposición de la materia orgánica, la alteración de los minerales del suelo y los procesos pedogenéticos, así como también puede afectar el crecimiento vegetal a través de su efecto sobre la solubilidad de los nutrientes. Estudios realizados en la biosecuencia bosque, en el sudoeste de la provincia del Neuquén, a nivel de los primeros cinco centímetros del suelo, mostraron que el $P$. ponderosa no generó acidificación de la solución del suelo. Esta respuesta estuvo determinada por el alto poder tampón de los suelos alofánicos, consecuencia del dominio de cenizas holocénicas básicas como material parental y de los productos de su evolución bajo régimen de humedad del suelo údico (2). En la transición bosque-estepa, parte de los factores de formación del suelo son similares a los de la biosecuencia bosque y otros cambian. Presentan en común el material de origen de los suelos -cenizas volcánicas básicas-y se diferencian por el régimen hídrico -que pasa de údico a xérico- y por el tipo de vegetación arealmente dominante -que pasa de bosque mixto de Nothofagus a estepa subarbustivograminosa- $(3,4)$. La respuesta del suelo bajo $P$. ponderosa puede ser diferente en la transición bosque-estepa dadas las diferencias pedogenéticas entre ambos sistemas. Bajo régimen údico los suelos evolucionan a Andisoles y bajo régimen xérico comienza una cristalización de los productos de la alteración del material piroclástico, pudiendo evolucionar a Molisoles manteniendo parte de las propiedades de los Andisoles $(5,6,8)$. En este contexto, el presente estudio pretende evaluar la respuesta del suelo en cuanto a su reacción -acidez de la solución, acidez de intercambio, bases intercambiables, capacidad de intercambio y actividad del aluminio- a nivel de los primeros cinco centímetros de suelo mineral frente al cambio de vegetación producto de las forestaciones con $P$. ponderosa con relación a la vegetación de estepa subarbustivo-graminosa.

\section{MATERIAL Y METODOS}

El estudio se realizó en la región andinopatagónica, sudoeste de la provincia de Neuquén, región Extra Andina, subregión de Planicies, Colinas y Serranías, circunscrito a la biosecuencia transición bosque-estepa. El clima general es templado, subhúmedo, con estación seca contrastante, con precipitaciones medias anuales que disminuyen paulatinamente hacia el oeste de 1.000 a 500 $\mathrm{mm}(4,7)$ y vientos dominantes con dirección oeste-este.

En la transición entre el bosque de Nothofagus y la estepa patagónica la vegetación nativa arealmente dominante correspondió a la formación vegetal de estepa subarbustivo-graminosa asociada con bosquetes aislados de Nothofagus antarctica (Forst. f.) Oerst. (ñire) y Austrocedrus chilensis (Don.) Florín et Boutleje (ciprés de la cordillera) (3). Las especies dominantes fueron Stipa speciosa Trin. (coirón amargo) y Mulinum spinosum Pers. (neneo), acompañadas principalmente por Festuca pallescens (St. Yv.) Parodi (coirón dulce), Acaena splendens (acaena), Eryngium paniculatum Camb. et Domb. (cardoncillo), Oxalis valdiviense Barn. (vinagrillo), Rumex acetosella (vinagrillo), Senecio sp. (senecio) y otras, con 50 a $80 \%$ de cobertura. Las forestaciones con $P$. ponderosa correspondieron a la primera plantación, con edades comprendidas entre los 14 y 19 años al momento de este estudio y con 80 a $100 \%$ de cobertura de la tierra.

El criterio utilizado para delimitar los extremos de la biosecuencia transición bosque-estepa se basó en los procesos que ocurren en los suelos 
a medida que el régimen hídrico de los suelos varía de údico a xérico (oeste) y de xérico a arídico (este). El estudio estuvo restringido a suelos derivados de cenizas volcánicas por ser aquellos con mayor potencial forestal (8) y a la estepa subarbustivo-graminosa por ser la formación vegetal dominante del área. El diseño de muestreo se realizó tomando como base la metodología usada por Broquen et al. $(1,2)$ y Candan et al. (9). Se utilizaron los doce sitios seleccionados por Broquen et al. (1), ubicados en un transverso con dirección aproximada oeste-este, desde los $39^{\circ} 57^{\prime}$ $\mathrm{S}, 71^{\circ} 11^{\prime} \mathrm{O}$ (Estancia Collunco) hasta los $39^{\circ} 59^{\prime}$ $\mathrm{S}, 71^{\circ} 51^{\prime} \mathrm{O}$ (Estancia Huechahue) presentando cada sitio los dos tipos de vegetación. Se asumió que las diferencias observadas bajo los dos tipos de vegetación se asociaban solamente con la vegetación, estimándose que no existían diferencias iniciales. Esto se funda en que la selección de los sitios implicó la elección de unidades de muestreo que tuvieran un uso previo y condiciones ecológicas lo más similares posibles. Todos los suelos fueron bien drenados, con régimen hídrico xérico y térmico mésico, correspondiéndoles el orden Andisoles y Molisoles (10), con dominio de texturas gruesas y presencia de pumicita y lapilli en la parte superior del perfil y materiales más arcillosos en los horizontes subsuperficiales. La densidad aparente del suelo fue de media a baja (en promedio $1,13 \mathrm{Mg} \mathrm{m}^{-3}$ ) y el color en húmedo de negro (10YR 2/1) a pardo muy oscuro (10YR $2 / 2$ ) en superficie cambiando a pardo oscuro (10YR 3/3) en profundidad. Las secuencias de horizontes fueron muy semejantes entre los suelos bajo las dos condiciones de vegetación.

La forma de incorporación de la materia orgánica fue nítidamente diferente entre la vegetación nativa -estepa subarbustivo-graminosa (ESG)- y la implantada -bosque de $P$. ponderosa (PP)-. La vegetación de ESG incorpora materia orgánica principalmente a nivel de las raíces, mientras que el PP aporta principalmente a través del mantillo con una alta relación $\mathrm{C} / \mathrm{N}$ (11). Esto se reflejó claramente en la formación de un horizonte orgánico bajo PP y no bajo ESG (1), donde el mantillo también tiene una alta relación $\mathrm{C} / \mathrm{N}$ pero la cantidad aportada es mínima (12).

Dado que a nivel de perfiles de suelo no se han encontrado diferencias significativas en la densidad aparente, el $\mathrm{pH}\left(\mathrm{H}_{2} \mathrm{O}\right)$ y el $\mathrm{pH}(\mathrm{KCl})(9$, 13) y se han detectado cambios generados por el tipo de uso a nivel de los primeros centímetros
(2), en el presente trabajo se analizaron los primeros cinco centímetros de suelo mineral.

Las propiedades consideradas fueron aquellas relacionadas con la reacción del suelo y con la actividad del aluminio por ser suelos derivados de cenizas volcánicas. Éstas fueron: acidez de la solución del suelo, $\mathrm{pH}\left(\mathrm{H}_{2} \mathrm{O}\right)$; acidez de intercambio, $\mathrm{pH}(\mathrm{KCl}) ; \Delta \mathrm{pH}$ definido como la diferencia entre el $\mathrm{pH}\left(\mathrm{H}_{2} \mathrm{O}\right)$ y el $\mathrm{pH}(\mathrm{KCl})$; $\mathrm{pH}(\mathrm{NaF})$; bases de cambio; capacidad de intercambio catiónico (CIC); capacidad de intercambio catiónico efectiva (CICE) y la retención de fosfato. La presencia del aluminio activo en suelos puede ser detectada midiendo potenciométricamente el aumento del pH en una solución molar de NaF después de dos minutos de reacción con el suelo. Cuando el valor de $\mathrm{pH}(\mathrm{NaF})$ a los 2' es mayor a 9,2 se considera que indica la presencia de aluminio activo (14). Por otra parte, el $\mathrm{pH}(\mathrm{NaF})$ a los 2' y 60' puede ser utilizado para inferir la mineralogía de las arcillas dominantes. Cuando ambos valores de $\mathrm{pH}(\mathrm{NaF})$ son mayores a 9,2 hay un dominio del alófano en el suelo, si ambos valores son menores a 9,2 hay un dominio de haloisita, y si a los 2' es menor a 9,2 y a los 60' es mayor a 9,2 indica un dominio de imogolita (15).

Debido a las múltiples dificultades para estimar correctamente la CIC en suelos derivados de cenizas volcánicas y la variabilidad de los valores obtenidos por los métodos convencionales se resolvió también determinar la CICE, definida como la suma de las bases totales más el $\mathrm{Al}^{3+}$ y el $\mathrm{H}^{+}$ extractable con $\mathrm{KCl}$ (16). Los análisis se hicieron acorde a los procedimientos presentados por Sadzawka (17).

Sintesis del diseño experimental. El tratamiento de los datos se realizó a través de la metodología propia de un experimento factorial aleatorizado considerando dos niveles de vegetación y cinco niveles de profundidad (18). Como ya se plantea$\mathrm{ra}$, se fijaron algunas restricciones a priori para las características de sitio de forma tal que todos los suelos fuesen derivados de cenizas volcánicas y con ambos tipos de vegetación en igual rango de altitud, gradiente, orientación, posición y adyacentes, con una distancia entre $20 \mathrm{~m}$ y $30 \mathrm{~m}$ de separación.

La edad de las plantaciones se determinó por lectura de anillos de tarugos extraídos con barreno de incremento a los $0,30 \mathrm{~m}$. Para realizar el muestreo bajo PP se sortearon aleatoriamente tres 
árboles, descartando el borde. Se fijó el lugar del muestreo a una distancia de $50 \mathrm{~cm}$ del tronco del árbol donde se removió el horizonte orgánico hasta llegar al contacto con el primer horizonte mineral. El procedimiento se repitió bajo los tres árboles, obteniendo una muestra compuesta por tres submuestras de cada centímetro en los primeros cinco centímetros por parcela, utilizando una plancheta diseñada para tal efecto. La distancia al tronco fijada para el muestreo se estableció acorde con algunos antecedentes $(2,19,20)$ que muestran que el área de influencia de la copa es donde se han encontrado cambios significativos. Para realizar el muestreo bajo ESG se utilizó el mismo criterio que bajo PP tomando la distancia a $50 \mathrm{~cm}$ del centro del arbusto o mata.

A partir de datos obtenidos anteriormente $(8$, $9,13)$ y de un relevamiento y muestreo piloto, se analizaron los estadísticos sencillos de los valores de $\mathrm{pH}\left(\mathrm{H}_{2} \mathrm{O}\right)$ del horizonte superficial bajo ESG. Estos estadísticos se utilizaron para determinar la variación de la variable a analizar y el número mínimo de muestras necesarias $(\alpha=0,05)$. El $\mathrm{pH}\left(\mathrm{H}_{2} \mathrm{O}\right)$ varió entre un valor mínimo de 6,1 y un valor máximo de 7,6, con una media de 6,7 y un coeficiente de variación de 0,05 . Se calculó el "n" según Cochran (21) dando un valor de 4,43.

Se muestreó un total de veinticuatro parcelas, doce bajo vegetación natural de ESG y doce bajo vegetación implantada de PP, las que abarcaron una variación altitudinal entre los $1.030 \mathrm{~m}$ hasta los $740 \mathrm{~m}$, diferentes posiciones topográficas y un amplio rango de exposiciones, encontrándose un detalle de las características de los sitios en Broquen et al. (1). Las parcelas comprendieron un rango de precipitaciones medias anuales entre los $900 \mathrm{~mm}$ y los $690 \mathrm{~mm}$ (7). Se realizaron en total ciento veinte determinaciones para el $\mathrm{pH}\left(\mathrm{H}_{2} \mathrm{O}\right)$ y ciento veinte para el $\mathrm{pH}(\mathrm{KCl})$. Se realizó el análisis factorial considerando el $\mathrm{pH}\left(\mathrm{H}_{2} \mathrm{O}\right)$ y el $\mathrm{pH}(\mathrm{KCl})$ a dos niveles de vegetación -ESG y $\mathrm{PP}-$, cinco niveles de profundidad, con doce repeticiones. Al haber repeticiones se pudo analizar la interacción. Las determinaciones de CIC, CICE, bases de intercambio, $\mathrm{pH}(\mathrm{NaF})$ y retención de fosfato se hicieron en los primeros cinco centímetros en bloque dado que no hubo interacción con la profundidad para las otras variables relacionadas. Para evaluar la influencia del tiempo, se realizó el análisis de regresión entre aquellas propiedades cuya variación fuese significativa y la edad de las plantaciones.

\section{RESULTADOS Y DISCUSION}

En el cuadro 1 se presenta una síntesis de las condiciones de sitio (1) y de los valores medios de las propiedades fisicoquímicas analizadas correspondientes a las muestras de los primeros cinco centímetros del suelo mineral para cada parcela. En el cuadro 2 se presentan los estadísticos sencillos de las variables analizadas.

El valor promedio del $\mathrm{pH}\left(\mathrm{H}_{2} \mathrm{O}\right)$ en superficie bajo ESG fue 6,5 con un 95\% de probabilidad de encontrarse entre 6,40 y 6,59. Para PP el valor promedio fue 6,3 con un $95 \%$ de probabilidad de encontrarse entre 6,20 y 6,32 (cuadro 2). En promedio fueron suelos ligeramente ácidos en superficie (22) tanto bajo ESG como PP, abarcando el rango de moderadamente ácidos a neutros (cuadro 2). El poder tampón depende de los iones y moléculas en la solución del suelo y de los minerales y materia orgánica de la fracción sólida del suelo y su comportamiento varía según rangos de $\mathrm{pH}$ (23). El rango de $\mathrm{pH}\left(\mathrm{H}_{2} \mathrm{O}\right)$ medido dentro de los primeros cinco centímetros del suelo mineral indicaría que el $\mathrm{pH}$ de la solución del suelo estuvo regulado por el sistema $\mathrm{H}_{2} \mathrm{O} / \mathrm{CO}_{2}$ y que el mecanismo principal de tamponación de estos suelos estaría gobernado por el intercambio catiónico y los silicatos del suelo (23).

El valor medio del $\mathrm{pH}(\mathrm{KCl})$ bajo ESG fue 5,5 con un $95 \%$ de probabilidad de encontrarse entre 5,41 y 5,56 y bajo PP fue 5,1 con un $95 \%$ de probabilidad de encontrarse entre 5,07 y 5,19. Tanto el $\mathrm{pH}\left(\mathrm{H}_{2} \mathrm{O}\right)$ como el $\mathrm{pH}(\mathrm{KCl})$ fueron menores bajo PP que bajo ESG (cuadro 2, figura 1).

Para el $\mathrm{pH}\left(\mathrm{H}_{2} \mathrm{O}\right)$, el análisis de varianza mostró que no existió interacción entre tipo de vegetación y profundidad, por lo que se pudo pasar a analizar el efecto del tipo de vegetación encontrándose un descenso altamente significativo del $\mathrm{pH}\left(\mathrm{H}_{2} \mathrm{O}\right)$ bajo PP con relación a la ESG sin que presente diferencias significativas con la profundidad (cuadro 3, figura 1).

De lo analizado se desprende que el suelo se acidificó a nivel de los primeros cinco centímetros, pasando de un valor promedio de 6,5 a 6,3 , lo que no implicó un cambio en la clase de acidez. Respecto al rango de valores, los mismos fueron algo menores bajo PP pero corresponden al mismo rango de clases de acidez, el que abarca de moderadamente ácidos a neutros (cuadro 1). 


\section{CUADRO 1}

Principales características del sitio y suelo bajo vegetación de estepa subarbustivo-graminosa (ESG) y $P$. ponderosa (PP), valores promedio en los primeros $5 \mathrm{~cm}$ del suelo en cada parcela.

Main site and soil characteristics under shrub-gramineous steppe vegetation (ESG) and Ponderosa pine (PP), average values for the first $5 \mathrm{~cm}$ for each plot.

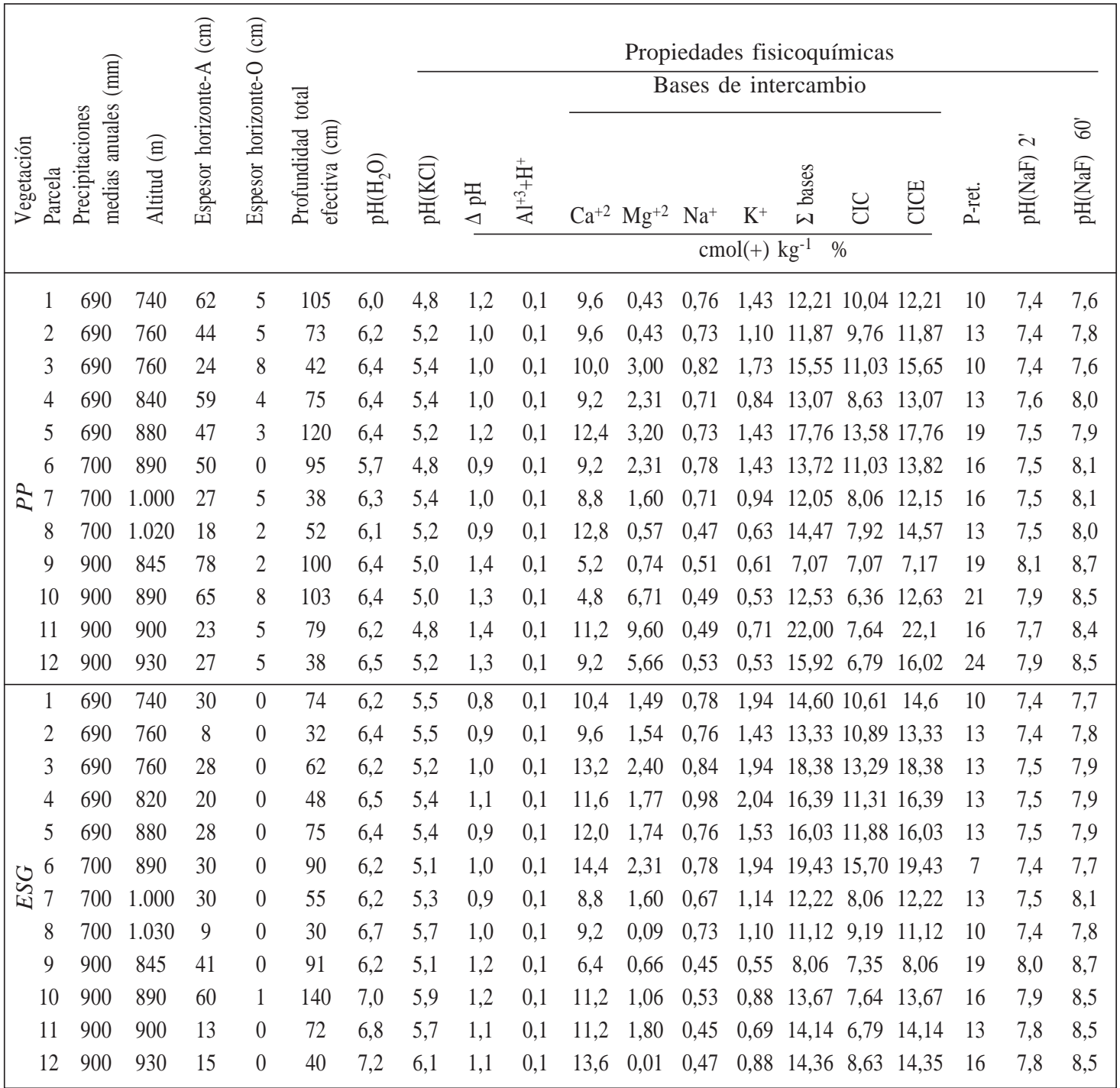

Ref.: $\Delta \mathrm{pH}$ : diferencia entre $\mathrm{pH}\left(\mathrm{H}_{2} \mathrm{O}\right)$ y $\mathrm{pH}(\mathrm{KCl}) ; \sum$ bases: total de bases de cambio; CIC: capacidad de intercambio catiónico; CICE: capacidad de intercambio catiónico efectiva; P-ret.: retención de fosfato. 


\section{CUADRO 2}

Estadísticos sencillos de los valores de $\mathrm{pH}\left(\mathrm{H}_{2} \mathrm{O}\right), \mathrm{pH}(\mathrm{KCl}), \Delta \mathrm{pH}\left[\mathrm{pH}\left(\mathrm{H}_{2} \mathrm{O}\right)-\mathrm{pH}(\mathrm{KCl})\right], \mathrm{pH}(\mathrm{NaF})$, retención de fosfato (P-ret.), total de bases intercambiables ( $\Sigma$ bases), capacidad de intercambio catiónico (CIC), en los primeros $5 \mathrm{~cm}$ para la totalidad de las parcelas bajo vegetación de estepa subarbustivo-graminosa (ESG) y $P$. ponderosa $(\mathrm{PP})$.

Single statistics for $\mathrm{pH}\left(\mathrm{H}_{2} \mathrm{O}\right), \mathrm{pH}(\mathrm{KCl}), \Delta \mathrm{pH}\left[\mathrm{pH}\left(\mathrm{H}_{2} \mathrm{O}\right)-\mathrm{pH}(\mathrm{KCl})\right], \mathrm{pH}(\mathrm{NaF})$, phosphate retention (P-ret.), total exchangeable bases ( $\Sigma$ bases), cation exchange capacity (CIC) values, in the first $5 \mathrm{~cm}$, for all plots under shrubgramineous steppe vegetation (ESG) and Ponderosa pine (PP).

\begin{tabular}{|c|c|c|c|c|c|c|c|c|c|c|c|c|c|c|}
\hline & \multicolumn{2}{|c|}{$\mathrm{pH}\left(\mathrm{H}_{2} \mathrm{O}\right)$} & \multicolumn{2}{|c|}{$\mathrm{pH}(\mathrm{KCl})$} & \multicolumn{2}{|c|}{$\Delta \mathrm{pH}$} & \multicolumn{2}{|c|}{$\mathrm{pH}(\mathrm{NaF}) 2^{\prime}$} & \multicolumn{2}{|c|}{$\begin{array}{c}\text { P-ret. } \\
\%\end{array}$} & \multicolumn{3}{|c|}{$\begin{array}{l}\Sigma \text { bases } \\
\quad \mathrm{cmol}(+) \mathrm{kg}^{-1}\end{array}$} & CIC \\
\hline & ESG & PP & ESG & PP & ESG & PP & ESG & PP & ESG & PP & ESG & PP & ESG & PP \\
\hline Media & 6,5 & 6,3 & 5,5 & 5,1 & 1,0 & 1,1 & 7,6 & 7,6 & 13 & 16 & 14,36 & 14,02 & 10,11 & 8,99 \\
\hline Desvío estándar & 0,365 & 0,244 & 0,302 & 0,233 & 0,147 & 0,187 & 0,228 & 0,229 & 3,133 & 4,324 & 3,092 & 3,659 & 2,676 & 2,142 \\
\hline Mínimo & 5,9 & 5,6 & 5,0 & 4,7 & 0,6 & 0,8 & 7,4 & 7,4 & 7 & 10 & 8,06 & 7,07 & 8,91 & 7,22 \\
\hline Máximo & 7,3 & 6,7 & 6,1 & 5,5 & 1,3 & 1,5 & 8,1 & 8,0 & 19 & 24 & 19,43 & 22,00 & 6,79 & 6,36 \\
\hline $\mathrm{n}$ & 60 & 60 & 60 & 60 & 60 & 60 & 12 & 12 & 12 & 12 & 12 & 12 & 12 & 12 \\
\hline
\end{tabular}

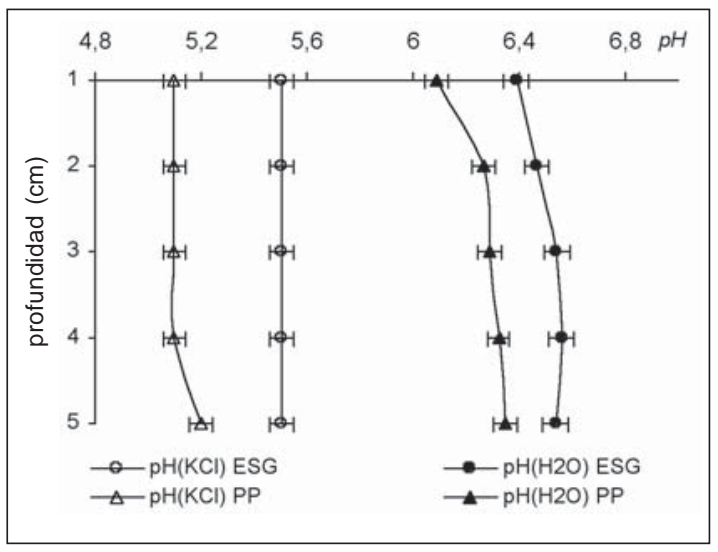

Figura 1. Valores medios por centímetro del $\mathrm{pH}\left(\mathrm{H}_{2} \mathrm{O}\right)$ y $\mathrm{pH}(\mathrm{KCl})$ en los primeros $5 \mathrm{~cm}$ para la totalidad de las parcelas bajo vegetación de estepa subarbustivograminosa (ESG) y $P$. ponderosa $(\mathrm{PP})$.

$\mathrm{pH}\left(\mathrm{H}_{2} \mathrm{O}\right)$ and $\mathrm{pH}(\mathrm{KCl})$ average values per centimeter in the first $5 \mathrm{~cm}$ for all plots under shrub-gramineous steppe vegetation (ESG) and Ponderosa pine (PP).

A partir del análisis de varianza para el $\mathrm{pH}(\mathrm{KCl})$ (cuadro 4) se pudo afirmar que ocurrió un descenso altamente significativo del $\mathrm{pH}(\mathrm{KCl})$ bajo PP con relación a la ESG. El comportamiento del sistema a nivel de los primeros $5 \mathrm{~cm}$ respecto al $\mathrm{pH}\left(\mathrm{H}_{2} \mathrm{O}\right)$ y $\mathrm{pH}(\mathrm{KCl})$ fue diferente que a nivel del perfil del suelo donde no se detectaron cambios $(9,13)$.
El descenso del $\mathrm{pH}(\mathrm{KCl})$ fue mayor que el de $\mathrm{pH}\left(\mathrm{H}_{2} \mathrm{O}\right)$, lo que se reflejó en un incremento de la diferencia entre ambos $-\Delta \mathrm{pH}-$. El $\Delta \mathrm{pH}$ indica la presencia de cargas variables (24) y al ser este valor positivo (cuadros 1,5 y 6 ) se podría esperar un dominio en superficie de cargas electronegativas, mayor bajo PP que bajo vegetación de ESG. Este incremento de cargas negativas podría deberse a diferencias en el tipo de materia orgánica incorporada por PP. Por otra parte, los valores del $\Delta \mathrm{pH}$ indican que los suelos tendrían una alta acidez de intercambio, la que aumentó bajo PP.

Con el análisis de varianza para el $\Delta \mathrm{pH}$ tampoco se encontró una interacción significativa entre los factores, siendo la diferencia altamente significativa $(\mathrm{ESG}<\mathrm{PP})$. Esto indicaría que ocurrió un incremento de la acidez intercambiable y que probablemente ocurrió un aumento de la electronegatividad de los coloides en los primeros cinco centímetros del suelo. No existió tampoco en este caso interacción del $\Delta \mathrm{pH}$ con la profundidad.

$\mathrm{Al}$ ser suelos derivados de cenizas volcánicas el incremento de la acidez intercambiable podría deberse a un incremento del aluminio de intercambio y en menor grado con el aluminio activo. $\mathrm{El} \mathrm{pH}(\mathrm{NaF})$ fue en todos los casos menor a 9,2 a los 2' y 60' (cuadros 1 y 2), indicando baja actividad de aluminio. Los valores medios de $\mathrm{pH}(\mathrm{NaF})$ fueron iguales bajo ambos tipos de vegetación (2': 7,$6 ; 60$ ': 8,1), por lo que no habría incidencia del 


\section{CUADRO 3}

Análisis de varianza: variable dependiente $\mathrm{pH}\left(\mathrm{H}_{2} \mathrm{O}\right)$, dos niveles de vegetación y cinco de profundidad.

Variance analysis: dependent variable $\mathrm{pH}\left(\mathrm{H}_{2} \mathrm{O}\right)$, two vegetation levels and five depth levels.

\begin{tabular}{|crccrrr|}
\hline $\begin{array}{c}\text { Fuente } \\
\text { de variación }\end{array}$ & $\begin{array}{c}\text { Suma de } \\
\text { cuadrados }\end{array}$ & $\begin{array}{c}\text { Grados de } \\
\text { libertad }\end{array}$ & Cuadrado medio & F & Probabilidad & $\begin{array}{c}\text { F crítico } \\
\alpha=0,05\end{array}$ \\
\hline Profundidad & 0,70011 & 4 & 0,17503 & 1,81 & 0,1317 & 2,45 \\
Vegetación & 1,67088 & 1 & 1,67088 & 17,29 & 0,0001 & 3,93 \\
Interacción & 0,04795 & 4 & 0,01120 & 0,12 & 0,9735 & 2,45 \\
Error & 110,04879 & 110 & 0,11476 & & & \\
Total corregido & 13,04879 & 119 & & & & \\
\hline
\end{tabular}

\section{CUADRO 4}

Análisis de varianza: variable dependiente $\mathrm{pH}(\mathrm{KCl})$, dos niveles de vegetación y cinco de profundidad. Variance analysis: dependent variable $\mathrm{pH}(\mathrm{KCl})$, two vegetation levels and five depth levels.

\begin{tabular}{|ccccccc|}
\hline $\begin{array}{c}\text { Fuente } \\
\text { de variación }\end{array}$ & $\begin{array}{c}\text { Suma de } \\
\text { cuadrados }\end{array}$ & $\begin{array}{c}\text { Grados de } \\
\text { libertad }\end{array}$ & Cuadrado medio & F & Probabilidad & $\begin{array}{r}\text { F crítico } \\
\alpha=0,05\end{array}$ \\
\hline Profundidad & 0,06286 & 4 & 0,01571 & 0,20 & 0,9357 & 2,45 \\
Vegetación & 3,90602 & 1 & 3,90602 & 50,67 & 0,00006 & 3,93 \\
Interacción & 0,03349 & 4 & 0,08373 & 0,10 & 0,9792 & 2,45 \\
Error & 8,47926 & 110 & 0,07708 & & & \\
Total corregido & 12,4816 & 119 & & & & \\
\hline
\end{tabular}

\section{CUADRO 5}

Análisis de varianza: variable dependiente $\Delta \mathrm{pH}$, dos niveles de vegetación y cinco de profundidad. Variance analysis: dependent variable $\Delta \mathrm{pH}$, two vegetation levels and five depth levels.

\begin{tabular}{|ccccccc|}
\hline $\begin{array}{c}\text { Fuente } \\
\text { de variación }\end{array}$ & $\begin{array}{c}\text { Suma de } \\
\text { cuadrados }\end{array}$ & $\begin{array}{c}\text { Grados de } \\
\text { libertad }\end{array}$ & Cuadrado medio & F & Probabilidad & $\begin{array}{c}\text { F crítico } \\
\alpha=0,05\end{array}$ \\
\hline Profundidad & 0,3751 & 4 & 0,0934 & 3,50 & 0,0099 & 2,45 \\
Vegetación & 0,4675 & 1 & 0,4675 & 17,45 & 0,0001 & 3,93 \\
Interacción & 0,0150 & 4 & 0,0038 & 0,14 & 0,97 & 2,45 \\
Error & 2,9467 & 110 & 0,0268 & & & \\
Total corregido & 3,80439 & 119 & & & & \\
\hline
\end{tabular}

tipo de vegetación sobre el aluminio activo. Según Irisarri (15) estos valores de $\mathrm{pH}(\mathrm{NaF})$ implican un dominio de haloisita, lo que sería consecuencia de los procesos que ocurren en la evolución de suelos de cenizas volcánicas bajo régimen xérico donde los compuestos amorfos producto de la alteración del vidrio y las cenizas volcánicas no evolucionan a alófano e imogolita sino a arcillas embrionarias con un grado mayor de cristalización y con menor poder tampón $(5,8,16)$, lo que se acentúa con menores precipitaciones medias anuales (cuadro 1, figura 3 ). 


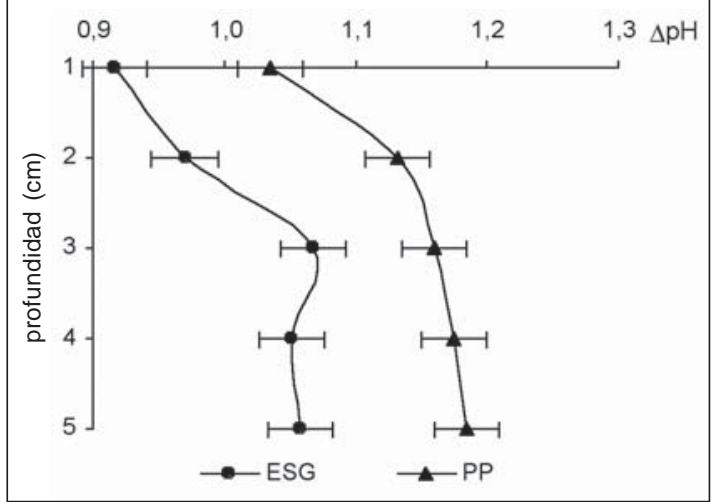

Figura 2. $\Delta \mathrm{pH}\left[\mathrm{pH}\left(\mathrm{H}_{2} \mathrm{O}\right)-\mathrm{pH}(\mathrm{KCl})\right]$ promedio por centímetro en los primeros $5 \mathrm{~cm}$ para la totalidad de las parcelas bajo vegetación de estepa subarbustivograminosa (ESG) y $P$. ponderosa $(\mathrm{PP})$.

$\Delta \mathrm{pH}\left[\mathrm{pH}\left(\mathrm{H}_{2} \mathrm{O}\right)-\mathrm{pH}(\mathrm{KCl})\right]$ average values per centimeter in the first $5 \mathrm{~cm}$ for all plots under shrub-gramineous steppe vegetation (ESG) and Ponderosa pine (PP).

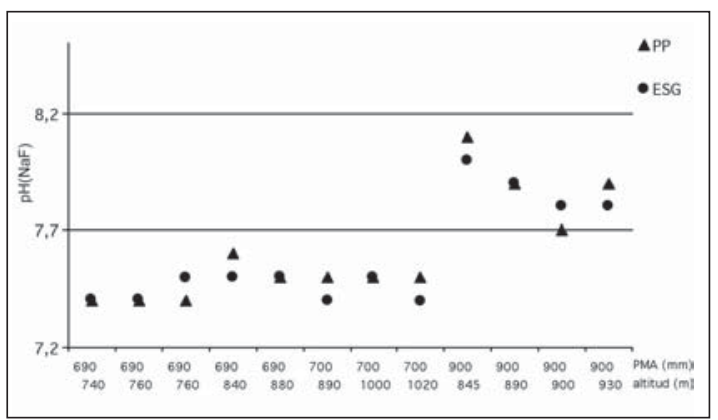

Figura 3. $\mathrm{pH}(\mathrm{NaF}) 2$ ' en las doce parcelas bajo vegetación de estepa subarbustivo-graminosa (ESG) y $P$. ponderosa (PP) ordenadas por precipitación media anual (mm) (PMA) y altitud (m).

$\mathrm{pH}(\mathrm{NaF}) 2^{\prime}$ in the twelve plots under shrub-gramineous steppe vegetation (ESG) and Ponderosa pine (PP) ordered by mean annual precipitation (PMA) and altitude (m).

Esta baja actividad del aluminio se refleja en los valores de retención de fosfato (cuadros 1 y 2), la que no varió bajo PP con relación a la ESG. Los valores medios encontrados fueron bajos (ESG: 16\%; PP: 13\%) si se considera que son suelos derivados de cenizas volcánicas, reflejando la incidencia del bajo nivel de alteración y dominio de vidrio volcánico en la fracción arena (6).

Los valores de las bases totales fueron medios (ESG: 14,32 cmol(+) kg-1; PP: 14,02 cmol(+) $\mathrm{kg}^{-1}$ ), acorde con los antecedentes para suelos de cenizas volcánicas bajo régimen hídrico xérico (8). La diferencia en el tenor de bases bajo ambas vegetaciones fue estadísticamente no significativa $(\mathrm{P}>0,05)$, lo que se visualiza en el diagrama de barras correspondiente al total de bases en cada unidad de muestreo bajo ambos tipos de vegetación (figura 4).

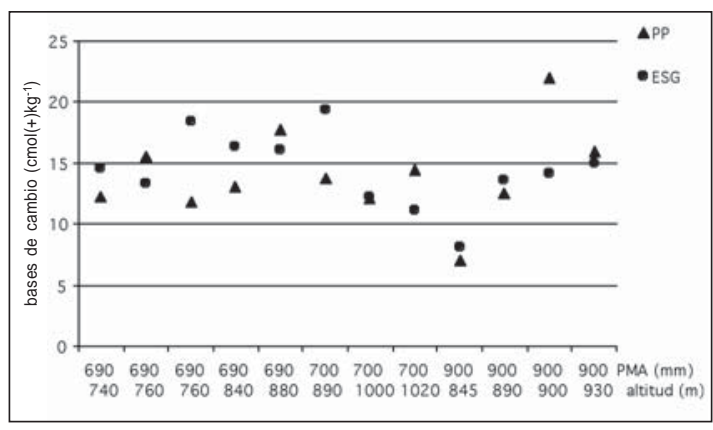

Figura 4. Contenido de bases de cambio totales en cada muestra de suelo de los primeros $5 \mathrm{~cm}$ de cada parcela, ordenadas por precipitación media anual $(\mathrm{mm})$ (PMA) y altitud (m), bajo vegetación de estepa subarbustivo-graminosa (ESG) y P. ponderosa (PP).

Total base content of each soil sample within the first five centimeters in each plot ordered by mean annual precipitation (mm) (PMA) and altitude (m), under shrub-gramineous steppe vegetation (ESG) and Ponderosa pine (PP).

Dado que las especies son diferentes, es importante considerar por una parte el total de bases y por otra las diferencias respecto a cada una de ellas. En la figura 5 se presenta gráficamente la distribución promedio del contenido de las diferentes bases bajo ambos tipos de vegetación y se observa que en ambos casos el Ca fue el que tuvo mayor peso (ESG: 77\%; PP: 67\%). Bajo PP se encontró una tendencia a que el $\mathrm{Ca}$ disminuyera acompañada de un incremento del magnesio (ESG: 9\%; PP: $20 \%$, diferencia significativa para $\alpha=0,10)$. Fue el magnesio el catión que presentó mayor variación entre ambos tipos de vegetación, ya que el potasio y el sodio se mantuvieron constantes. Esto podría indicar alguna diferencia en el ciclo de nutrientes entre PP y ESG, lo que debería ser estudiado en futuras investigaciones, no existiendo antecedentes en la región sobre la demanda específica de nutrientes por las especies de la estepa subarbustivo-graminosa (12).

La CIC no varió entre ambos tipos de vegetación (ESG: 9,93 cmol(+) kg-1; PP: 9,13 cmol(+) $\mathrm{kg}^{-1}$ ) y fue menor a lo esperable, lo que puede deberse a que son suelos de carga variable con dominio de vidrio poco alterado y reflejaron una 


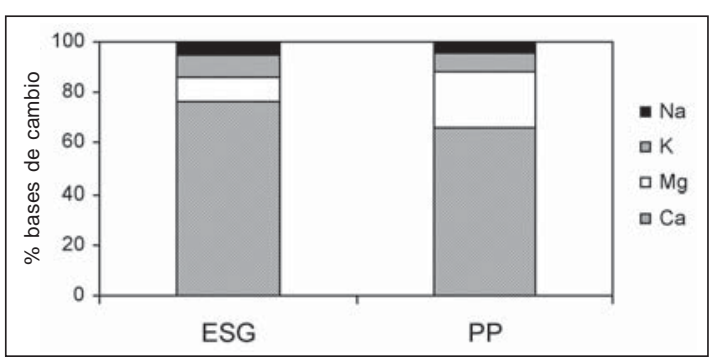

Figura 5. Contenido de $\mathrm{Ca}, \mathrm{Mg}, \mathrm{K}$ y $\mathrm{Na}$ expresado en \% bajo vegetación de estepa subarbustivo-graminosa (ESG) y $P$. ponderosa (PP) (cada valor corresponde al promedio de la totalidad de las muestras de los primeros cinco centímetros del suelo).

$\mathrm{Ca}, \mathrm{Mg}, \mathrm{K}$ and $\mathrm{Na}$ content expressed as \%, under shrubgramineous steppe vegetation (ESG) and Ponderosa pine (PP) (each value corresponds to the average values for all plots within the first five centimeters.

situación metodológica más que el comportamiento del suelo $(16,6)$. La CICE reflejó principalmente la variación en el contenido de bases totales ya que los valores de $\left(\mathrm{Al}^{3+}+\mathrm{H}^{+}\right)$fueron muy bajos, con un promedio de $0,10 \mathrm{cmol}(+) \mathrm{kg}^{-1}$ bajo ESG y PP (cuadro 1). Este resultado es acorde a los antecedentes presentados por Shoji et al. (24), quienes plantean que la acidez intercambiable es típicamente baja en suelos derivados de cenizas volcánicas. Tal como plantea Besoain (16): “...si el valor de CIC es variable en cuanto a las circunstancias experimentales, lo es también en cuanto a la especie misma, lo cual implica una doble contradicción y que la expresión cuantitativa tenga un valor restringido". El autor cita a Harada y Wada (1973), quienes señalan que la suma del $\mathrm{H}^{+}$ de cambio el $\mathrm{Al}^{+3}$ y las bases no pueden igualarse al de la CIC de los suelos alofánicos dado que desarrollan cargas positivas o negativas de acuerdo al $\mathrm{pH}$. De lo antedicho se desprende que los valores de CIC y saturación en bases obtenidos reflejan una situación metodológica más que el comportamiento del suelo, por lo que la interpretación de los resultados fue limitada y no permitió inferir el comportamiento en cuanto a la capacidad de intercambio catiónico de estos suelos, siendo fundamental continuar con estudios en esta temática. No se detectó tampoco variación del $\mathrm{pH}(\mathrm{NaF})$, lo que indica que no existió un incremento de la actividad del aluminio, reflejándose la incidencia de las precipitaciones medias anuales en el tenor del aluminio activo bajo ambos tipos de vegetación (figura 3).
Se presenta, a modo de síntesis, un cuadro comparativo de las variables estudiadas a nivel de los cinco primeros centímetros del suelo mineral bajo ESG y bajo PP (cuadro 6).

El relevamiento de antecedentes y lo informado por los productores indicaron que el PP se estableció por sustitución directa de la ESG sin ningún otro uso intermedio más que el ganadero, correspondiendo todas a primera plantación cuya instalación fue realizada sin fertilizaciones, riego o quemas. Por lo que, dadas las condiciones de sitio equivalentes de las parcelas bajo ambos tipos de vegetación, las diferencias encontradas pudieron atribuirse al cambio de vegetación.

Se encontraron evidencias que indican que el sistema bajo PP se acidificó a nivel de los primeros cinco centímetros respecto a la situación bajo vegetación de ESG sin que ocurra una variación en la clase de acidez del suelo. El incremento de la acidez intercambiable no fue acompañado por un incremento del aluminio intercambiable, por lo que tampoco se estarían generando problemas de toxicidad por aluminio.

Para explicar los procesos mediante los cuales el PP pudo determinar un descenso del $\mathrm{pH}$, asumiendo que no hubo cambios en la aireación y en los procesos redox, es fundamental considerar el conjunto de mecanismos involucrados en la acidificación del suelo. Estos involucran (i) el tiempo transcurrido desde el momento en que se produce el cambio de vegetación; (ii) el rango de $\mathrm{pH}\left(\mathrm{H}_{2} \mathrm{O}\right)$ de la solución del suelo; (iii) el incremento de la saturación ácida del complejo de intercambio o disminución de la saturación básica y (iv) el incremento de los ácidos almacenados en el suelo, particularmente la materia orgánica humificada y la acumulación de humus más fuertemente acídico.

(i) El análisis de regresión entre el descenso del $\mathrm{pH}\left(\mathrm{H}_{2} \mathrm{O}\right)$ y la edad de las plantaciones no permitió establecer un patrón de comportamiento que permita explicar el cambio en la acidez con el tiempo transcurrido después de 14 años desde la instalación de las plantaciones (edad vs $\left.\mathrm{pH}\left(\mathrm{H}_{2} \mathrm{O}\right) \mathrm{R}^{2}=0,3018\right)$. Este análisis se realizó también con respecto a la acidez intercambiable, encontrándose el mismo comportamiento. Esto podría indicar que la acidificación del suelo a nivel de los $5 \mathrm{~cm}$ ocurrió en un período relativamente corto, probablemente menor a 14 años y que no continuó acidificándose dado que tampoco se encontró una clara tendencia a que la 


\section{CUADRO 6}

Comparación entre las propiedades del suelo en los primeros $5 \mathrm{~cm}$ bajo vegetación de estepa subarbustivo-graminosa (ESG) y $P$. ponderosa $(\mathrm{PP})$.

Comparative table between soil properties within the first $5 \mathrm{~cm}$ under shrub-gramineous steppe vegetation (ESG) and Ponderosa pine (PP).

\begin{tabular}{|c|c|c|}
\hline & PP & ESG \\
\hline $\mathrm{pH}\left(\mathrm{H}_{2} \mathrm{O}\right)$ & $\begin{array}{c}6,3^{* *} \\
\Rightarrow \text { varió la acidez de la solución del suelo } \\
\Rightarrow \text { no cambió la clase de acidez: } \\
\text { ligeramente ácidos }\end{array}$ & 6,5 \\
\hline $\mathrm{pH}(\mathrm{KCl})$ & $\begin{array}{c}5,1^{* *} \\
\Rightarrow \text { dominado por el sistema } \mathrm{H}_{2} \mathrm{O} / \mathrm{CO}_{2} \\
\Rightarrow \text { mecanismo de tamponación regulado por } \\
\text { bases de cambio y mineralogía de las arcillas } \\
\Rightarrow \text { incrementó la acidez de cambio bajo PP }\end{array}$ & 5,5 \\
\hline$\Delta \mathrm{pH}$ & $\begin{array}{c}1,1^{* *} \\
\Rightarrow \text { electronegativos } \\
\Rightarrow \text { incrementaría la electronegatividad bajo PP }\end{array}$ & 1,0 \\
\hline $\begin{array}{l}\mathrm{pH}(\mathrm{NaF}) 2^{\prime} \\
\mathrm{pH}(\mathrm{NaF}) 60^{\prime}\end{array}$ & $\begin{array}{c}7,6 \\
8,1 \\
\Rightarrow \text { se mantuvo, no hubo incremento del Al activo } \\
\Rightarrow \text { determinado por la evolución de la ceniza } \\
\text { volcánica con régimen xérico }\end{array}$ & $\begin{array}{l}7,6 \\
8,1\end{array}$ \\
\hline P- retención & $\begin{array}{c}16 \\
\Rightarrow \text { se mantuvo bajo PP }\end{array}$ & 13 \\
\hline $\begin{array}{l}\Sigma \text { Bases de cambio } \\
\left.\quad\left(\mathrm{cmol}^{(+)}\right) \mathrm{kg}^{-1}\right) \\
\mathrm{Ca}^{2+}\left(\mathrm{cmol}(+) \mathrm{kg}^{-1}\right) \\
\mathrm{Mg}^{2+}\left(\mathrm{cmol}(+) \mathrm{kg}^{-1}\right)\end{array}$ & $\begin{array}{c}14 \\
9 \\
3^{*} \\
\Rightarrow \text { Ca dominó el complejo de cambio } \\
\Rightarrow \text { se mantuvo el total de bases en superficie } \\
\Rightarrow \text { tendió a aumentar el Mg y a disminuir el Ca bajo PP }\end{array}$ & $\begin{array}{c}14 \\
11 \\
1\end{array}$ \\
\hline & $\begin{array}{c}\Rightarrow \text { suelo con bajo poder de amortiguación } \\
\Rightarrow \text { incrementó la acidez de la solución bajo PP } \\
\Rightarrow \text { incrementó acidez intercambiable bajo PP }\end{array}$ & \\
\hline
\end{tabular}

acidez incremente con el paso del tiempo, lo que se deberá corroborar con posteriores monitoreos. Los resultados serían acordes a lo informado por Binkley (25), quien plantea que la manifestación del cambio en la acidez por la influencia de especies arbóreas ocurre en pocos años o en algo más que algunas décadas.

(ii) La acidificación estaría condicionada por el rango de $\mathrm{pH}$ en que se encuentran estos suelos que, como ya se planteara, corresponde a un rango de tamponación por bases de cambio y por los silicatos cristalinos (23). Los resultados obtenidos así como los antecedentes $(4,5,8,15,24)$ indican un bajo tenor de compuestos amorfos y un posible dominio de compuestos paracristalinos así como de haloisita como productos de la meteorización del material parental bajo régimen xérico. La CIC de las arcillas paracristalinas sería menor que la del alófano y baja para la haloisita (10 a $\left.50 \mathrm{cmol}(+) \mathrm{kg}^{-1}\right)(16)$. $\mathrm{Al}$ ser suelos con un escaso desarrollo y con bajo 
tenor de arcillas cristalinas su poder tampón sería menor que cuando se desarrollan bajo régimen údico. La incidencia del poder tampón del aluminio también fue baja dado por el bajo tenor en la alofanización, lo que fue acompañado por una baja actividad del aluminio $(\mathrm{pH}(\mathrm{KCl})>5$ y $\mathrm{pH}(\mathrm{NaF})<$ 9,2 ). Esto indica que el material parental y su evolución tuvieron una fuerte incidencia en el efecto del PP sobre las propiedades del suelo, lo que es acorde a lo informado por Augusto et al. (26), quienes plantean que el efecto de las especies arbóreas en cuanto a su impacto en la tasa de acidificación fue menor en comparación con otros factores y con fuerte influencia de la naturaleza del material parental del suelo.

(iii) El incremento de la acidez de la solución del suelo así como el de la acidez intercambiable no fueron acompañados por una disminución en el contenido total de bases de cambio ni por un incremento del aluminio intercambiable, por lo que no se puede atribuir la acidificación bajo PP a la desaturación del complejo de intercambio.

(iv) La dinámica de la materia orgánica puede determinar cambios en la reacción del suelo tanto en lo que se refiere a la acidez de la solución del suelo como a la acidez intercambiable y su grado de manifestación depende de los mecanismos de tamponación. Estos definen reacciones químicas específicas que resisten cambios debidos al ingreso de $\mathrm{H}^{+}$al sistema, así como controlan la actividad de los $\mathrm{H}^{+}$en la solución del suelo (23). El bajo poder tampón de estos suelos determinaría que los protones aportados por la ionización de los grupos funcionales incorporados $(-\mathrm{COOH}$, $\mathrm{OH},-\mathrm{NH}_{2}$ ) por la materia orgánica de $\mathrm{PP}$ se hayan manifestado en el descenso del $\mathrm{pH}\left(\mathrm{H}_{2} \mathrm{O}\right)$, lo que no ocurre bajo régimen údico según lo informado por Broquen et al. (2). Por otra parte, la materia orgánica ejerce un alto poder amortiguador a cualquier $\mathrm{pH}>4$ (27), por lo que probablemente también haya ocurrido un descenso en el contenido de materia orgánica y un cambio en el tipo de materia húmica con incremento de grupos funcionales ionizables. Ambos supuestos deberán ser considerados en futuros estudios.

\section{CONCLUSIONES}

Por debajo del horizonte-O, en los primeros cinco centímetros del suelo mineral, comenzaron a manifestarse alteraciones generadas por el cambio de vegetación. Las variables analizadas no mostraron interacción con la profundidad, no existiendo evidencias de la incidencia de algún factor que determine una variación espacial en sentido vertical dentro de los primeros cinco centímetros del suelo mineral para las variables analizadas.

La acidez de la solución del suelo incrementó en los primeros cinco centímetros del suelo mineral bajo $P$. ponderosa con relación a la vegetación de estepa subarbustivo-graminosa, sin determinar un cambio en la clase de acidez del suelo, manteniéndose en promedio como ligeramente ácidos.

La acidez intercambiable aumentó en los primeros cinco centímetros del suelo mineral bajo $P$. ponderosa con relación a la vegetación de estepa subarbustivo-graminosa.

Ambos descensos fueron consecuencia del bajo poder tampón de los suelos determinado por la evolución del material de origen -cenizas volcánicas básicas- bajo régimen xérico.

El total de bases de cambio no varió bajo $P$. ponderosa con relación a la vegetación de estepa subarbustivo-graminosa, tendiendo a disminuir el calcio y a incrementar el magnesio bajo $P$. ponderosa, lo que sería un indicio de un cambio en el ciclo de los nutrientes minerales.

Dado que no cambió la clase de acidez, no disminuyó el total de bases intercambiables y no aumentó la retención de fosfato bajo $P$. ponderosa, se puede afirmar que al momento de la realización de este estudio, el grado de acidificación de los primeros cinco centímetros del suelo mineral no generaría variaciones relevantes en las condiciones del suelo para la germinación y crecimiento tanto de especies autóctonas como de futuras forestaciones.

La acidificación e incremento de la acidez intercambiable observados, aunque no consisten en un cambio drástico de las propiedades del suelo, son indicadores de cambios que comienzan a ocurrir en el suelo desde la instalación de las forestaciones, debiendo considerar su uso en futuros monitoreos.

\section{BIBLIOGRAFIA}

(1) BROQUEN P., G. FALBO, A. APCARIAN, F. CANDAN, J. L. GIRARDIN, V. PELLEGRINI. Forestaciones y erosión en la transición bosque-estepa (Andinopatagonia, Argentina). Invest. Agrar.: Sist. Recur. For. (España), 2003, vol. 12 , $\mathrm{N}^{\circ} 2$, p. $99-110$. 
(2) BROQUEN P., F. CANDAN, G. FALBO, A. APCARIAN, J. L. GIRARDIN. Efecto del Pinus ponderosa Dougl. sobre la reacción del suelo en el sudoeste del Neuquén, Argentina. Bosque (Chile), 2002, vol. 23, No 1, p. 47-59.

(3) MOVIA C., G. OWER, C. PEREZ. Estudio de la vegetación natural. Provincia del Neuquén. Argentina: Ministerio de Economía y Hacienda, Subsecretaría de Recursos Naturales, Provincia del Neuquén, 1982, 149 p.

(4) FERRER J., J. IRISARRI, M. MENDIA. Estudio Regional de Suelos de la Provincia del Neuquén. vol. 1. tomo 2, Argentina: CFI - COPADE - Provincia del Neuquén. 1990. Síntesis de los factores del medio geográfico y de las propiedades de los suelos, $147 \mathrm{p}$.

(5) COLMET DAAGE F., A. MARCOLIN, C. LOPEZ, M. LANCIOTTI, J. AYESA, D. BRAN, E. ANDENMATTEN, P. BROQUEN, J. L. GIRARDIN, G. CORTES, J. IRISARRI, E. BESOAIN, A. SADZAWKA, G. SEPULVEDA, S. MASSARO, G. MILLOT, P. BOULEOU. Zonificación del potencial forestal de la cordillera y precordillera Transecta San Martín de los Andes. Paso Hua Hum - Collon Cura. Argentina: INTAORSTOM, 1988, $167 \mathrm{p}$.

(6) BROQUEN P., F. CANDAN, G. FALBO. Suelos derivados de cenizas volcánicas en la bioclimosecuencia bosque-transición a la estepa. Actas del XVIII Congreso Argentino de la Ciencia del Suelo, 2002, p. 110.

(7) BARROS, V., V. CORDON, C. L. MOYANO, R. J. MENDEZ, J. C. FORQUERA, O. PIZZIO. Cartas de precipitaciones del oeste de las provincias de Río Negro y Neuquén. Primera contribución. Argentina: UNCo CENPAT-CONICET, 1983, 100 p.

(8) COLMET DAAGE, F., A. MARCOLIN, C. LOPEZ, M. LANCIOTTI, J. AYESA, D. BRAN, E. ANDENMATTEN, P. BROQUEN, J. L. GIRARDIN, G. CORTES, J. IRISARRI, E. BESOAIN, A. SADZAWKA, G. SEPULVEDA, S. MASSARO, G. MILLOT, P. BOULEOU. Características de los suelos derivados de cenizas volcánicas de la cordillera y precordillera del norte de la Patagonia. Argentina: INTA-ORSTOM, 1988, 39 p.

(9) CANDAN F., P. BROQUEN, G. FALBO. Propiedades químicas de un Vitrixerand húmico y un Haploxerol vitrándico bajo pino ponderosa y estepa (SO Neuquén). Revista de Investigaciones Agropecuarias (Argentina), 2003, vol. 32, $\mathrm{N}^{\mathrm{o}} 1$, p. 71-88.

(10) SOIL SURVEY STAFF. Keys to Soil Taxonomy. Technical Monograph $\mathrm{N}^{\text {o }} 19.5^{\text {th }}$ ed., USA: AID-USDA, Pocahontas Press Inc. Blacksburg Virginia, 1992, 591 p.

(11) KONONOVA, M. Materia orgánica del suelo. $1^{\mathrm{a}}$ ed., Barcelona: Oikos Tau, 1982, 365 p.
(12) MAZZARINO M. J., M. BERTILLER, T. SCHLICHTER, M. GOBBI. Nutrient cycling in Patagonian ecosystems. Ecología Austral (Argentina), 1998, vol. 8, p. 167-181.

(13) BROQUEN P., J. L. GIRARDIN, M. C. FRUGONI. Evaluación de algunas propiedades de los suelos derivados de cenizas volcánicas asociados a forestaciones con coníferas exóticas. R.A. Bosque (Chile), 1995, vol. 16, $\mathrm{N}^{\mathrm{o}}$ 2, p. 69-79.

(14) SAIGUSA M., S. SHOJI, M. OTOWA. Clay mineralogy of Andisols showing a hydrosequence and its relationships to their physical and chemical properties. Pedologist (Japan), 1991, vol. 35, $\mathrm{N}^{\circ}$ 1, p. 21-33.

(15) IRISARRI J. A. La propuesta de reclasificación de los Andepts de Argentina, de acuerdo al Orden Andisoles. Actas del Taller Soil Taxonomy. Argentina: INTAAICET-AACS, 2000, p. 18-27.

(16) BESOAIN, E. Mineralogía de Arcillas de Suelos. Costa Rica: IICA. 1985. Aluminosilicatos no cristalinos y paracristalinos del suelo, p. 533-543.

(17) SADZAWKA, A. Métodos de Análisis de Suelos. Serie Platina $N^{o}$ 16. Chile: INIA. 1990. 139 p.

(18) MONTGOMERY, D. C. Diseño y análisis de experimentos. $3^{a}$ ed., México: Grupo Ed. Iberoamérica, 1991. $538 \mathrm{p}$.

(19) ZINKE J.P. The pattern of influence of individual forest trees on soil properties. Ecology (USA), 1962, vol. 43, $\mathrm{N}^{\circ}$ 1, p. 130-133.

(20) DUCHAUFOUR, P. Atlas ecológico de los suelos del mundo. $1^{\text {a }}$ ed., Barcelona: Masson, 1977, 178 p.

(21) COCHRAN, W. Técnicas de muestreo. $2^{\mathrm{a}}$ ed., México: CECSA, 1977, $507 \mathrm{p}$.

(22) SOIL SURVEY STAFF. Soil Survey Manual. Handbook $\mathrm{N}^{\mathrm{o}} 18.5^{\text {th }}$ ed, USA: USDA, 1993, $436 \mathrm{p}$.

(23) ROBARGE W. P., D. W. JOHNSON. The effects of acidic deposition on forested soils. Adv. In Agron. (USA), 1992, vol. 47, p. 1-83.

(24) SHOJI, S., M. NANZYO, R. DAHLGREN. Volcanic ash soils. Genesis, Properties and Utilization. Amsterdam: Elsevier, 1993, 288 p.

(25) BINKLEY D. The influence of tree species on forest soils: processes and patterns. Trees and Soil. Workshop Proceedings. (New Zealand), 1994, p. 1-23.

(26) AUGUSTO L., J. P. BONNAUD, J. RANGER. Impact of tree species on forest soil acidification. Forest Ecology and Management (Amsterdam), 1998, vol. 105, $\mathrm{N}^{\circ} 1-3$, p. 67-78.

(27) TAN, K. H. Humic Matter in soil and the environment, principles and controversies. $1^{\mathrm{a}}$ ed., New York: Marcel Dekker, Inc., 2003, 325 p. 